\title{
S65-03
}

\section{NEUROBIOLOGICAL EFFECTS OF OPIOIDS - DATA FROM BRAIN IMAGING}

T. Kienast, A. Heinz, J. Wrase

Campus Charité Mitte; University Medical Center Berlin, Germany, Berlin, Germany

Neuroimaging provides insight into the main biological mechanisms underlying drug and alcohol dependence. Cardinal symptoms of drug dependence are the development of tolerance against drug effects, withdrawal symptoms, drug craving, reduced control over drug intake and harmful consequences of drug consumption. Brain imaging studies support the hypothesis that tolerance development can be understood as a neuroadaptive mechanism to ensure homeostasis during chronic drug intake. When drug consumption is suddenly interrupted during detoxification, the loss of homeostasis can manifest as a withdrawal syndrome. While tolerance development reduces the effects of chronic drug intake, sensitization is a neuroadaptive process that increases the effects of a drug dose. Brain imaging studies indicate that sensitisation and drug craving are associated with neuroadaptive processes in the brain reward system. This lecture gives a short review about the neurobiological background of opioid addiction 